\title{
Directing Single-Molecule Emission with DNA Origami-Assembled Optical Antennas
}

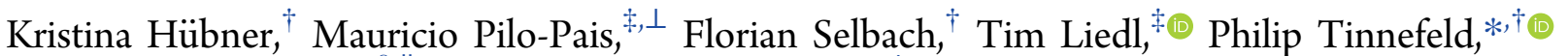 \\ Fernando D. Stefani, ${ }^{*}, \|_{\odot}$ and Guillermo P. Acuna ${ }^{*}, \perp^{(0)}$ \\ ${ }^{\dagger}$ Department of Chemistry and Center for NanoScience, Ludwig-Maximilians-Universität München, Butenandtstr. 5-13 Haus E, \\ 81377 München, Germany \\ ${ }^{\ddagger}$ Faculty of Physics and Center for NanoScience, Ludwig-Maximilians-Universität München, Geschwister-Scholl-Platz 1, 80539 \\ München, Germany \\ ${ }^{\S}$ Centro de Investigaciones en Bionanociencias (CIBION), Consejo Nacional de Investigaciones Científicas y Técnicas \\ (CONICET), Godoy Cruz 2390, C1425FQD, Ciudad Autónoma de Buenos Aires, Argentina \\ "Departamento de Física, Facultad de Ciencias Exactas y Naturales, Universidad de Buenos Aires, Güiraldes 2620, C1428EHA, \\ Ciudad Autónoma de Buenos Aires, Argentina \\ ${ }^{\perp}$ Department of Physics, University of Fribourg, Chemin du Musée 3, Fribourg CH-1700, Switzerland
}

Supporting Information

ABSTRACT: We demonstrate the capability of DNA selfassembled optical antennas to direct the emission of an individual fluorophore, which is free to rotate. DNA origami is used to fabricate optical antennas composed of two colloidal gold nanoparticles separated by a predefined gap and to place a single Cy5 fluorophore near the gap center. Although the fluorophore is able to rotate, its excitation and far-field emission is mediated by the antenna, with the emission directionality following a dipolar pattern according to the antenna main resonant mode. This work is intended to set out the basis for manipulating the emission pattern of single molecules with selfassembled optical antennas based on colloidal nanoparticles.

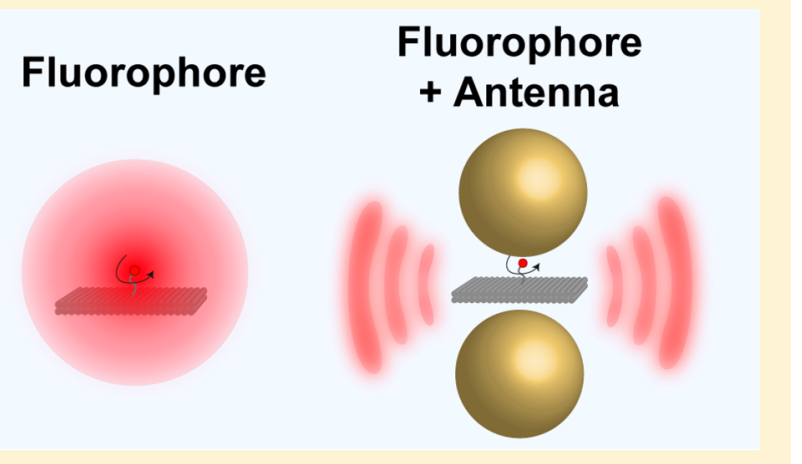

Fluorophore

Antenna

KEYWORDS: plasmonics, nanophotonics, metallic nanoparticles, DNA origami, optical antennas
$\mathrm{O}$ ptical antennas (OAs) ${ }^{1}$ represent the counterparts of radio- and microwave antennas within the visible spectrum. Essentially, OAs are built from metallic nanoparticles (NPs) whose localized surface plasmon resonances enable the control of light fields at the nanoscale. ${ }^{2}$ OAs can be engineered to manipulate the photophysical behavior of single photon emitters such as organic fluorophores or quantum dots placed in their vicinity. ${ }^{3}$ Pioneering examples are the "bow tie" OAs used to demonstrate enhancement of the fluorescence intensity of organic dyes placed at the hotspot between the gold elements ${ }^{4}$ and the monopole, and "Yagi-Uda" OAs used to demonstrate directionality in the emission of single emitters. $^{5-7}$ These examples share the fabrication approach based on electron beam lithography. Generally, top-down nanofabrication techniques such as e-beam lithography or ion beam milling offer great geometrical design versatility, but they also exhibit shortcomings. They are serial, which limits their throughput. The 3D fabrication and organization of (antenna) elements is difficult and limited to some degree of rotation of the sample with respect to the beam. There exist limitations to the quality and number of materials that can be used and combined. As a result, attaining OAs made of monocrystalline elements, as well as combining different materials, is challenging. Finally, it is virtually impossible to position single photon emitters with controlled stoichiometry in the near-field of the OAs with nanometer precision. ${ }^{1}$

The advent of the DNA origami technique ${ }^{8}$ opened up new pathways for nanophotonics ${ }^{9-16}$ as colloidal metallic NPs could be self-assembled in a parallel manner to form OAs. Furthermore, single photon emitters could be positioned in the near-field of OAs with nanometer precision and stoichiometric control. Following this approach, the performance of OAs could be revisited at the single-molecule level with higher geometrical control and more robust statistics, ${ }^{17-20}$ including their influence on the photophysical behavior of single photon emitters, such as the electronic transition rates, ${ }^{21}$ photostability, $^{22}$ fluorescence resonance energy transfer (FRET), ${ }^{23-25}$ surface-enhanced Raman scattering, ${ }^{26-28}$ strong coupling, $^{29}$ and super-resolution localization. ${ }^{30}$

Received: July 15, 2019

Revised: August 20, 2019 
DNA origami has turned out to be extremely efficient to organize NPs on nanometric geometries with high degree of positional and orientational control. ${ }^{31,32}$ The situation is different for single photon emitters. Whereas their position can be controlled well, orienting them over predefined directions in DNA origami remains an open challenge. ${ }^{33-36}$ In order to incorporate single fluorophores to DNA origami structures, they are attached to the backbone or a base of a short single-stranded DNA, hereafter termed "staple", which later binds to its complementary sequence on the DNA scaffold strand. One fluorophore can be bound to one or two staples through single or double linkers, respectively. ${ }^{37}$ Fluorophores integrated into DNA origami structures can exhibit a variety of behaviors, from free to rotate over fixed in an undefined orientation to confined in orientation, depending on their coupling chemistry, molecular structure, charge, and immediate surrounding environment. ${ }^{23}$

In this contribution, we investigate the emission directivity of rotating single fluorophores coupled to OAs. Cy5 fluorophores incorporated to DNA origami structures as shown in Figure 1A, which are able to rotate, do not present any directionality in excitation or emission. In contrast, in the presence of a dipolar $\mathrm{OA}$, both their excitation and emission are enhanced and become directional according to the antenna mode.

A schematic of the OA-Cy5 fluorophore system is shown Figure 1A. A two-layered, rectangular DNA origami sheet with a size of $\sim 50 \mathrm{~nm} \times 60 \mathrm{~nm} \times 5 \mathrm{~nm}$ is used to accommodate two ultrasmooth spherical Au NPs ${ }^{38}$ with a diameter of $60 \mathrm{~nm}$, each one at opposite sides of the origami. At approximately the center of the DNA origami structure, a single Cy5 fluorophore is incorporated at the $3^{\prime}$ end of a DNA single strand (see inset in Figure 1A). In this way, the single $\mathrm{Cy} 5$ molecule is located near the center of the $13 \mathrm{~nm}$ gap between the two Au NPs and is able to rotate when in solution. Figure $1 \mathrm{~B}$ shows exemplary TEM images of the dimer OAs illustrating the quality of our structures. The absorption and emission spectra of the Cy5 fluorophore employed together with a numerical simulation of the absorption and scattering cross section of the $\mathrm{OA}$ are included in Figure 1C. The OAs were self-assembled in solution. For fluorescence measurements, OAs were immobilized on a glass coverslip previously functionalized with BSAbiotin, neutravidin, and biotinylated single-stranded DNA complementary to the single-stranded DNA on the Au NP surface. As a result, OAs are expected to lie flat with their interparticle (main) axis parallel to the substrate surface. All optical measurements were performed in buffer (see Supporting Information for sample preparation and measurements details).

Functionalized NPs are mixed in high excess to the DNA origami structure to maximize the yield of dimer OAs. Gel electrophoresis permits to separate the desired structure from unbound NPs and any other unintentionally formed species resulting in a solution containing close to $100 \%$ of the target dimer structure. Figure 1D shows the distinct fluorescence lifetimes of the reference, monomer, and dimer samples obtained from single-molecule traces (details on the timeresolved single-molecule fluorescence measurements are given in the SI). As expected, the interaction between the fluorophores and the Au NPs reduces the fluorescence lifetime, with a more pronounced effect for dimer structures. ${ }^{17}$ The excited state lifetime of the $\mathrm{Cy} 5$ in the origami sheet is reduced from 1.7 to $0.6 \mathrm{~ns}$ when one $\mathrm{Au} \mathrm{NP}$ is attached, and to $0.2 \mathrm{~ns}$
A

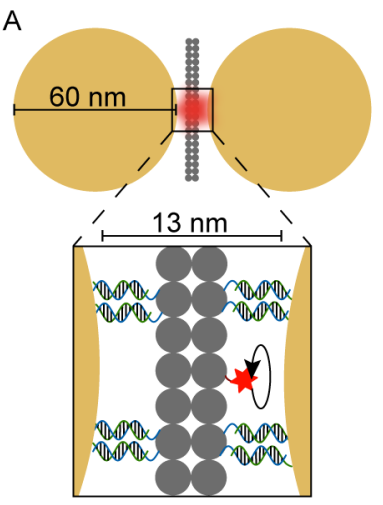

B

C
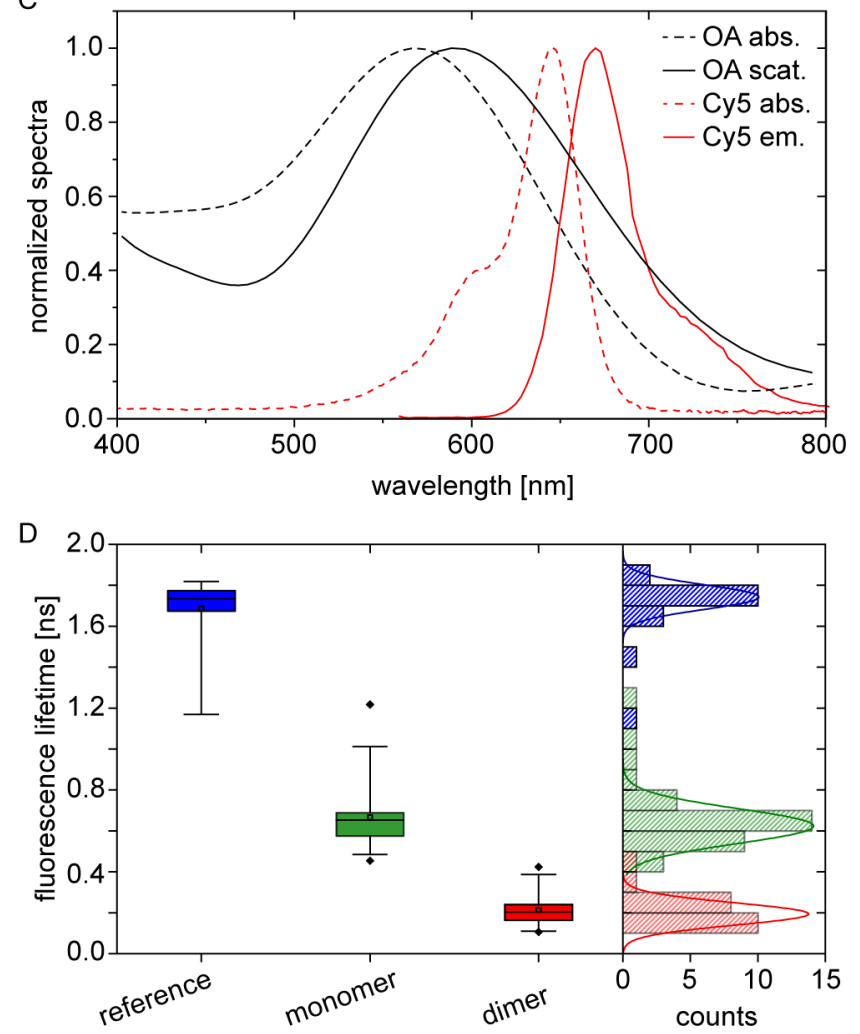

Figure 1. (A) Sketch of the OA-Cy5 structure composed of two gold NPs self-assembled onto a rectangular DNA origami. The inset depicts a close-up of the NP gap where the single Cy5 fluorophore is incorporated. (B) TEM images of the dimer structures (scale bar is $200 \mathrm{~nm}$ ). (C) Absorption and emission spectra of Cy5 together with the absorption and scattering cross section of the OA dimer. (D) Fluorescence lifetime histogram of samples containing two NPs (dimers), one NP (monomers), and the reference structure without NPs.

(limited by the instrumental response) when the dimer OA is formed. The relatively sharp lifetime distributions and TEM images reflect the quality of the preparation and the purity of the samples used in this study.

Two different single-molecule fluorescence measurements were performed on each of the individual OAs in order to determine the directionality imposed by the $\mathrm{OA}$ on the excitation and on the emission of the single fluorophores. The directionality of the emission was determined by wide-field defocused imaging. ${ }^{39}$ The directionality of the excitation was probed by monitoring the fluorescence intensity while rotating 
the direction of polarization of a linearly polarized laser used for excitation.

The fluorescence emission of molecules close to a planar interface has been extensively studied. ${ }^{39,40}$ The angular emission pattern depends strongly on the orientation of the molecular emission dipole with respect to the interface. In Figures $2 \mathrm{~A}-\mathrm{C}$, we include simulations for the emission patterns

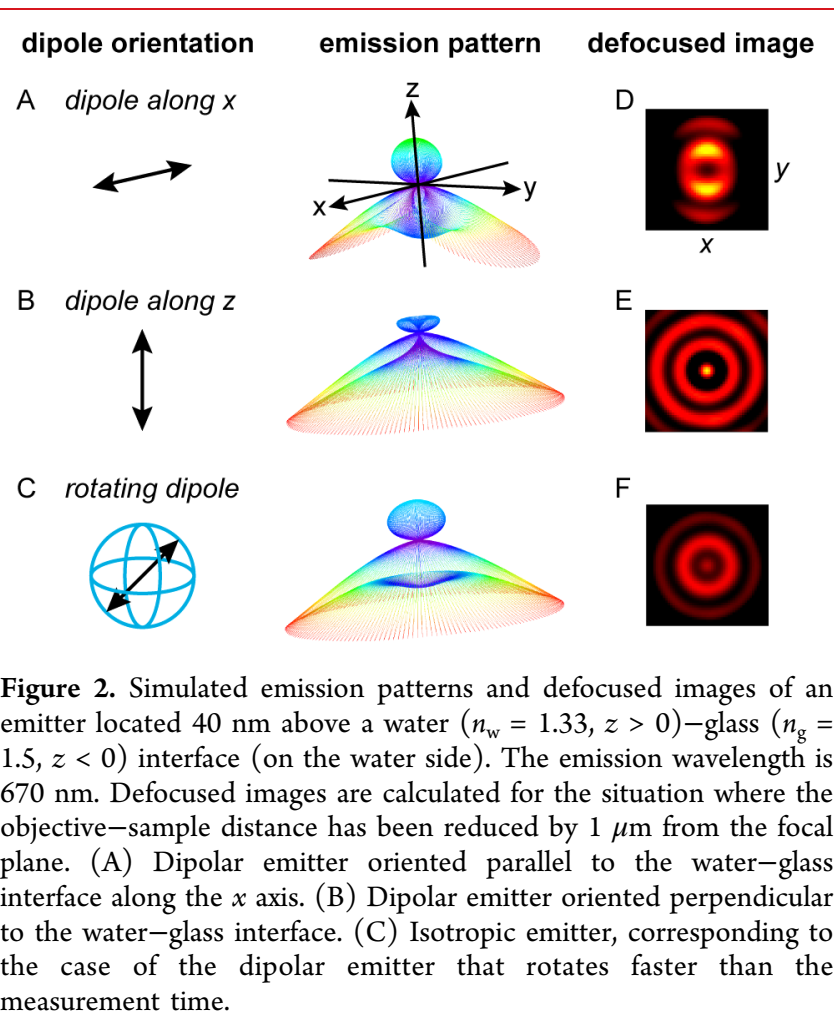

of a parallel, perpendicular and free to rotate $\mathrm{Cy} 5$ molecule placed $40 \mathrm{~nm}$ above the water-glass interface (on the water half-space). In all cases molecules emit preferentially into the glass half-space and at angles close to the critical angle of total internal reflection, but with distinct angular emission patterns. While the emission pattern of perpendicular molecules has rotational symmetry with respect to the surface normal, the pattern of a parallel molecule has two lobes separated by a gap along the dipole direction. For a fluorophore free to rotate, the emission pattern corresponds to the isotropic average of dipolar patterns with all possible orientations and has thus radial symmetry. Defocused imaging is a way to access experimentally the angular emission pattern of single molecules. ${ }^{39,41}$ Figures $2 \mathrm{D}-\mathrm{F}$ include the calculated defocused images of molecules oriented parallel, perpendicular, and freely rotating when reducing the objective-sample distance by $1 \mu \mathrm{m}$ from the focal plane.

Figure 3 shows typical defocused images $(\sim 1 \mu \mathrm{m})$ of both, Cy5 fluorophores conjugated to the center of the DNA origami platform with and without OAs. The rotational symmetry of Cy5 samples without OAs demonstrates that the Cy5 fluorophores are able to rotate on a time scale faster than the image acquisition time. Remarkably, the emission patterns of Cy5 molecules change qualitatively when they are coupled to the dimer OAs. In this case, the rotational symmetry is lost, and all detected emission patterns present the two lobes characteristic of an in-plane dipole. Each individual pattern of an $\mathrm{OA}-\mathrm{Cy} 5$ structure has a different in-plane orientation. In the presence of a dipolar OA, a rotating fluorophore operating at frequencies below the OA's resonance ${ }^{2,42}$ will couple to the resonant antenna mode when aligned parallel to the antenna's main axis. ${ }^{22,43}$ Under this orientation, the emission is expected to be enhanced and directional with a dipolar pattern. ${ }^{44}$ In contrast, for a perpendicular orientation, the fluorophore's radiative rate can be significantly suppressed leading to a negligible emission into the far-field. ${ }^{45-47}$ This behavior becomes intuitive when picturing the interactions between the $\mathrm{Cy} 5$ and its image charges produced on the NPs ${ }^{48}$ (Figure 3C). A perpendicularly oriented dipole is canceled out by its image dipole (Figure 3C-I), whereas a parallel oriented dipole is reinforced (Figure 3C-II). Therefore, despite the fact that the single $\mathrm{Cy} 5$ fluorophore is able to rotate, the presence of the OA will enhance and mediate the fluorophore's emission when its orientation is parallel to the main OA axis and suppress it when perpendicular. This is confirmed by numerical simulations of the fluorophore's quantum yield for the two orientations depicted in Figure 3C (see Figure S3). For each defocused pattern in Figure $3 \mathrm{~B}$, we extract $\theta_{e m}$ defined as the main in-plane emission angle.

Next, we studied the excitation directionality with the polarization-resolved excitation measurements. For each single structure, as the ones shown on Figure 3, fluorescent transients were extracted while rotating the incident light polarization. Exemplary transients are included in Figure 4A, where fluorescence enhancement (FE) refers to the fluorescence intensity normalized to the average fluorescence intensity of the reference structures (without NPs). The incident light
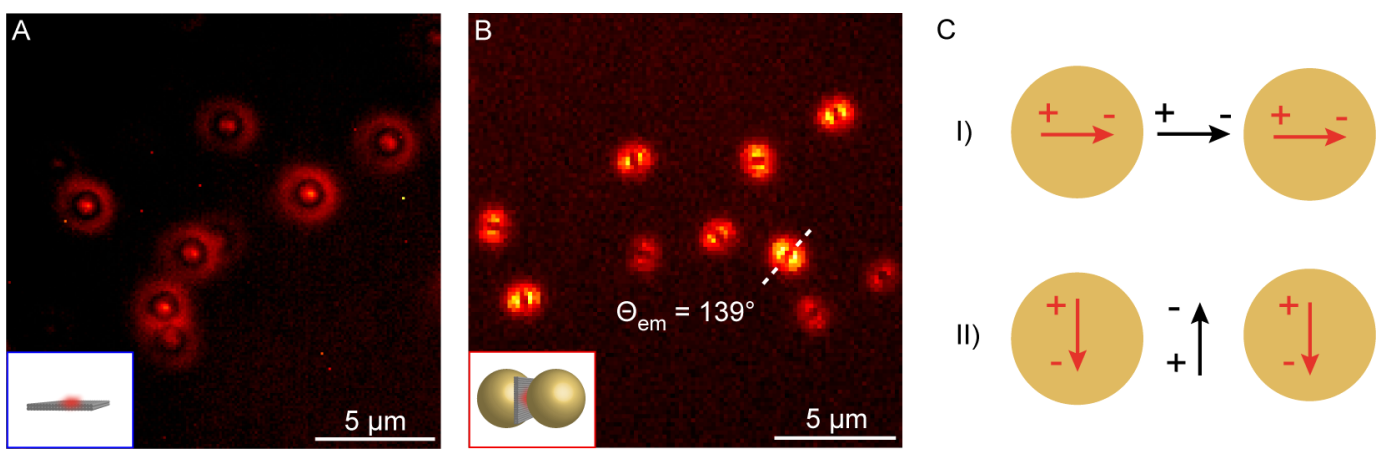

Figure 3. Defocused images of (A) the Cy5 reference sample and (B) the OA-Cy5 structures. The in-plane emission angle $\theta_{e m}$ is highlighted for a single OA. (C) Sketch of the image charges induced by a fluorophore on the OA elements. The black arrows represent the fluorophore's emission dipole moment, whereas the red arrows represent the induced dipoles in the NPs. 


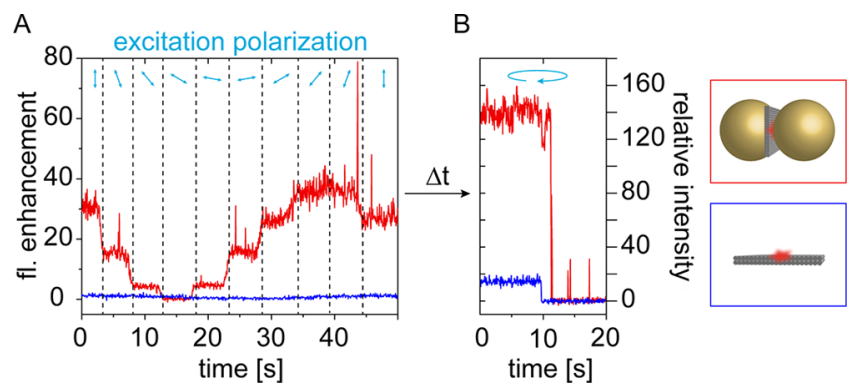

Figure 4. (A) Transients of fluorescence intensity for varying polarization angle of the excitation for the reference (no NP, blue) and OAs (NP dimer, red). The OA intensity is normalized to the average of the reference intensity and thus represents the fluorescence enhancement ( $\mathrm{FE})$. During the first $50 \mathrm{~s}$, the incident polarization is rotated. (B) Afterwards, the incident light power is increased and its polarization is turned circular in order to bleach the fluorophores to verify a single-step photobleaching.

polarization angle is rotated by $20^{\circ}$, sweeping a range of $180^{\circ}$. Finally, the fluorophores are photobleached using increased laser power and circularly polarized light to prove single-step bleaching behavior (Figure 4B). For the reference structures, fluorescence transients show negligible variations with the incident polarization angle. This is in line with our previous observation that $\mathrm{Cy} 5$ is able to rotate faster than the integration time. In contrast, the transients of OA-Cy5 structures show a clear periodic dependence with the incident polarization angle. As expected for a dipolar dimer OA, the electric field enhancement reaches the highest value when the incident light is polarized along the antenna axis. ${ }^{49}$

From these measurements, we extracted the polarization angle of maximum excitation $\theta_{e x}$, which corresponds to the inplane orientation of each OA. We note that for our analysis we considered only OAs that showed a clear cosine square response to the polarization angle and a single-step photobleaching, assuring that we probed dipolar OAs with a single fluorophore.

Finally, we combined the results of the independent measurements displayed in Figures $3 \mathrm{~B}$ and $4 \mathrm{~A}$ in order to study the emission and excitation directionality of each OA. Figure 5 displays a scatter plot of $\theta_{e m}$ versus $\theta_{e x}$ for 147 randomly oriented dimer OAs with a single $\mathrm{Cy} 5$ fluorophore.

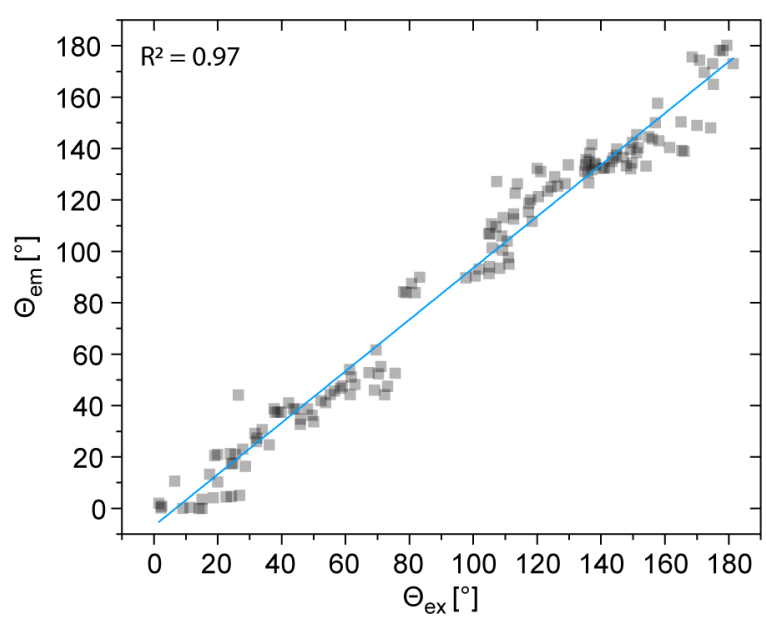

Figure 5. Scatter plot of the in-plane angles of emission $\theta_{e m}$ and excitation $\theta_{e x}$ for 147 individual OAs.
The strong correlation between both angles $\theta_{e m}$ and $\theta_{e x}$ confirms that the OAs impose directionality to the rotating fluorophores both on excitation and emission according to the antenna's main resonant mode.

In summary, using the DNA origami technique we selfassembled dipolar optical antennas made of two closely spaced gold nanoparticles with a single fluorescent molecule at their gap. By means of single-molecule measurements of the emission pattern and the polarization of maximum excitation, we showed that the excitation and emission of single fluorophores that are able to rotate can be made directional with optical antennas, according to the antenna's main resonant mode. These experiments provide a solid ground for more sophisticated photon routing experiments using single emitters and self-assembled optical antennas. ${ }^{50,51}$

\section{ASSOCIATED CONTENT}

\section{Supporting Information}

The Supporting Information is available free of charge on the ACS Publications website at DOI: 10.1021/acs.nanolett.9b02886.

Detailed information on gold nanoparticle fabrication, DNA origami folding, optical antenna assembly and immobilization, imaging systems, analysis, and numerical simulations (PDF)

\section{AUTHOR INFORMATION}

\section{Corresponding Authors}

*E-mail: philip.tinnefeld@cup.uni-muenchen.de (P.T.).

*E-mail: fernando.stefani@df.uba.ar (F.D.S.).

*E-mail: guillermo.acuna@unifr.ch (G.P.A.).

ORCID

Tim Liedl: 0000-0002-0040-0173

Philip Tinnefeld: 0000-0003-4290-7770

Fernando D. Stefani: 0000-0002-3277-7215

Guillermo P. Acuna: 0000-0001-8066-2677

Notes

The authors declare no competing financial interest.

\section{ACKNOWLEDGMENTS}

We would like to thank Mario Raab, Ija Jusuk, Florian Steiner, Jan Vogelsang, and Johann Bohlen for fruitful discussion. This work was supported by the Swiss National Science Foundation through the National Center of Competence in Research BioInspired Materials, with grants PICT2013-0792 and PICT2014-0739 of the Agencia Nacional de Promoción Cientifica y Tecnológica (ANPCYT, Argentina). P.T. and G.P.A. acknowledge support by the DFG (AC 279/2-1 and TI 329/9-1, INST 86/1904-1 FUGG, excellence cluster e-conversion). M.P. and T.L. thank the financial support from the European Research Council (ERC) through the starting grant 336440 (ORCA) and the DFG through the excellence cluster e-conversion. F.D.S. thanks the support of the Max-Planck-Society and the Alexander von Humboldt Foundation.

\section{REFERENCES}

(1) Novotny, L.; Van Hulst, N. Antennas for Light. Nat. Photonics 2011, 5 (2), 83-90.

(2) Biagioni, P.; Huang, J.-S.; Hecht, B. Nanoantennas for Visible and Infrared Radiation. Rep. Prog. Phys. 2012, 75 (2), 024402.

(3) Koenderink, A. F. Single-Photon Nanoantennas. ACS Photonics 2017, 4, 710-722. 
(4) Kinkhabwala, A.; Yu, Z.; Fan, S.; Avlasevich, Y.; Müllen, K.; Moerner, W. E. Large Single-Molecule Fluorescence Enhancements Produced by a Bowtie Nanoantenna. Nat. Photonics 2009, 3 (11), 654-657.

(5) Taminiau, T. H.; Stefani, F. D.; Segerink, F. B.; Van Hulst, N. F. Optical Antennas Direct Single-Molecule Emission. Nat. Photonics 2008, 2 (4), 234-237.

(6) Curto, A. G.; Volpe, G.; Taminiau, T. H.; Kreuzer, M. P.; Quidant, R.; Van Hulst, N. F. Unidirectional Emission of a Quantum Dot Coupled to a Nanoantenna. Science (Washington, DC, U. S.) 2010, 329 (5994), 930-933.

(7) Aouani, H.; Mahboub, O.; Devaux, E.; Rigneault, H.; Ebbesen, T. W.; Wenger, J. Large Molecular Fluorescence Enhancement by a Nanoaperture with Plasmonic Corrugations. Opt. Express 2011, 19 (14), 13056.

(8) Rothemund, P. W. K. Folding DNA to Create Nanoscale Shapes and Patterns. Nature 2006, 440, 297-302.

(9) Kuzyk, A.; Jungmann, R.; Acuna, G. P.; Liu, N. DNA Origami Route for Nanophotonics. ACS Photonics 2018, 5 (4), 1151-1163.

(10) Pilo-Pais, M.; Acuna, G. P.; Tinnefeld, P.; Liedl, T. Sculpting Light by Arranging Optical Components with DNA Nanostructures. MRS Bull. 2017, 42 (12), 936-942.

(11) Liu, N.; Liedl, T. DNA-Assembled Advanced Plasmonic Architectures. Chem. Rev. 2018, 118 (6), 3032-3053.

(12) Samanta, A.; Banerjee, S.; Liu, Y. DNA Nanotechnology for Nanophotonic Applications. Nanoscale 2015, 7 (6), 2210-2220.

(13) Lan, X.; Wang, Q. DNA-Programmed Self-Assembly of Photonic Nanoarchitectures. NPG Asia Mater. 2014, 6 (4), e97-e97.

(14) Wang, P.; Meyer, T. A.; Pan, V.; Dutta, P. K.; Ke, Y. The Beauty and Utility of DNA Origami. Chem. 2017, 2 (3), 359-382.

(15) Madsen, M.; Gothelf, K. V. Chemistries for DNA Nanotechnology. Chem. Rev. 2019. DOI: 10.1021/acs.chemrev.8b00570.

(16) Gopinath, A.; Miyazono, E.; Faraon, A.; Rothemund, P. W. K. Engineering and Mapping Nanocavity Emission via Precision Placement of DNA Origami. Nature 2016, 535 (7612), 401-405.

(17) Acuna, G. P.; Möller, F. M.; Holzmeister, P.; Beater, S.; Lalkens, B.; Tinnefeld, P. Fluorescence Enhancement at Docking Sites of DNA-Directed Self-Assembled Nanoantennas. Science (Washington, DC, U. S.) 2012, 338 (6106), 506-510.

(18) Prinz, J.; Heck, C.; Ellerik, L.; Merk, V.; Bald, I. DNA Origami Based $\mathrm{Au}-\mathrm{Ag}$-Core-Shell Nanoparticle Dimers with Single-Molecule SERS Sensitivity. Nanoscale 2016, 8 (10), 5612-5620.

(19) Simoncelli, S.; Roller, E.-M.; Urban, P.; Schreiber, R.; Turberfield, A. J.; Liedl, T.; Lohmüller, T. Quantitative SingleMolecule Surface-Enhanced Raman Scattering by Optothermal Tuning of DNA Origami-Assembled Plasmonic Nanoantennas. ACS Nano 2016, 10 (11), 9809-9815.

(20) Chikkaraddy, R.; Turek, V. A.; Kongsuwan, N.; Benz, F.; Carnegie, C.; van de Goor, T.; de Nijs, B.; Demetriadou, A.; Hess, O.; Keyser, U. F.; et al. Mapping Nanoscale Hotspots with SingleMolecule Emitters Assembled into Plasmonic Nanocavities Using DNA Origami. Nano Lett. 2018, 18 (1), 405-411.

(21) Holzmeister, P.; Pibiri, E.; Schmied, J. J.; Sen, T.; Acuna, G. P.; Tinnefeld, P. Quantum Yield and Excitation Rate of Single Molecules Close to Metallic Nanostructures. Nat. Commun. 2014, 5 (1), 5356.

(22) Kaminska, I.; Vietz, C.; Cuartero-González, Á.; Tinnefeld, P.; Fernández-Domínguez, A. I.; Acuna, G. P. Strong Plasmonic Enhancement of Single Molecule Photostability in Silver Dimer Optical Antennas. Nanophotonics 2018, 7 (3), 643-649.

(23) Aissaoui, N.; Moth-Poulsen, K.; Käll, M.; Johansson, P.; Wilhelmsson, L. M.; Albinsson, B. FRET Enhancement Close to Gold Nanoparticles Positioned in DNA Origami Constructs. Nanoscale 2017, 9 (2), 673-683.

(24) Vietz, C.; Lalkens, B.; Acuna, G. P.; Tinnefeld, P. Synergistic Combination of Unquenching and Plasmonic Fluorescence Enhancement in Fluorogenic Nucleic Acid Hybridization Probes. Nano Lett. 2017, 17 (10), 6496-6500.

(25) Bohlen, J.; Cuartero-González, Á.; Pibiri, E.; Ruhlandt, D.; Fernández-Domínguez, A. I.; Tinnefeld, P.; Acuna, G. P. Plasmon-
Assisted Förster Resonance Energy Transfer at the Single-Molecule Level in the Moderate Quenching Regime. Nanoscale 2019, 11 (16), 7674-7681.

(26) Thacker, V. V.; Herrmann, L. O.; Sigle, D. O.; Zhang, T.; Liedl, T.; Baumberg, J. J.; Keyser, U. F. DNA Origami Based Assembly of Gold Nanoparticle Dimers for Surface-Enhanced Raman Scattering. Nat. Commun. 2014, 5 (1), 3448.

(27) Prinz, J.; Schreiber, B.; Olejko, L.; Oertel, J.; Rackwitz, J.; Keller, A.; Bald, I. DNA Origami Substrates for Highly Sensitive Surface-Enhanced Raman Scattering. J. Phys. Chem. Lett. 2013, 4 (23), $4140-4145$.

(28) Kühler, P.; Roller, E. M.; Schreiber, R.; Liedl, T.; Lohmüller, T.; Feldmann, J. Plasmonic DNA-Origami Nanoantennas for SurfaceEnhanced Raman Spectroscopy. Nano Lett. 2014, 14 (5), 2914-2919.

(29) Ojambati, O. S.; Chikkaraddy, R.; Deacon, W. D.; Horton, M.; Kos, D.; Turek, V. A.; Keyser, U. F.; Baumberg, J. J. Quantum Electrodynamics at Room Temperature Coupling a Single Vibrating Molecule with a Plasmonic Nanocavity. Nat. Commun. 2019, 10 (1), 1049.

(30) Raab, M.; Vietz, C.; Stefani, F. D.; Acuna, G. P.; Tinnefeld, P. Shifting Molecular Localization by Plasmonic Coupling in a SingleMolecule Mirage. Nat. Commun. 2017, 8 (1), 13966.

(31) Pal, S.; Deng, Z.; Wang, H.; Zou, S.; Liu, Y.; Yan, H. DNA Directed Self-Assembly of Anisotropic Plasmonic Nanostructures. J. Am. Chem. Soc. 2011, 133 (44), 17606-17609.

(32) Zhan, P.; Wen, T.; Wang, Z.; He, Y.; Shi, J.; Wang, T.; Liu, X.; Lu, G.; Ding, B. DNA Origami Directed Assembly of Gold Bowtie Nanoantennas for Single-Molecule Surface-Enhanced Raman Scattering. Angew. Chem., Int. Ed. 2018, 57 (11), 2846-2850.

(33) de Torres, J.; Mivelle, M.; Moparthi, S. B.; Rigneault, H.; Van Hulst, N. F.; García-Parajó, M. F.; Margeat, E.; Wenger, J. Plasmonic Nanoantennas Enable Forbidden Förster Dipole-Dipole Energy Transfer and Enhance the FRET Efficiency. Nano Lett. 2016, 16 (10), 6222-6230.

(34) Kroutil, O.; Romancová, I.; Š́p, M.; Chval, Z. Cy3 and Cy5 Dyes Terminally Attached to 5 'C End of DNA: Structure, Dynamics, and Energetics. J. Phys. Chem. B 2014, 118 (47), 13564-13572.

(35) Boulais, E.; Sawaya, N. P. D.; Veneziano, R.; Andreoni, A.; Banal, J. L.; Kondo, T.; Mandal, S.; Lin, S.; Schlau-Cohen, G. S.; Woodbury, N. W.; et al. Programmed Coherent Coupling in a Synthetic DNA-Based Excitonic Circuit. Nat. Mater. 2018, 17 (2), $159-166$.

(36) Gopinath, A.; Thachuk, C.; Mitskovets, A.; Atwater, H. A.; Kirkpatrick, D.; Rothemund, P. W. K. Absolute and Arbitrary Orientation of Single Molecule Shapes. arXiv:1808.04544, 2018.

(37) Stennett, E. M. S.; Ma, N.; van der Vaart, A.; Levitus, M. Photophysical and Dynamical Properties of Doubly Linked Cy3-DNA Constructs. J. Phys. Chem. B 2014, 118 (1), 152-163.

(38) Yoon, J. H.; Selbach, F.; Langolf, L.; Schlücker, S. Ideal Dimers of Gold Nanospheres for Precision Plasmonics: Synthesis and Characterization at the Single-Particle Level for Identification of Higher Order Modes. Small 2018, 14 (4), 1702754.

(39) Böhmer, M.; Enderlein, J. Orientation Imaging of Single Molecules by Wide-Field Epifluorescence Microscopy. J. Opt. Soc. Am. B 2003, 20 (3), 554-559.

(40) Novotny, L.; Hecht, B. Principles of Nano-Optics, 2nd ed.; Cambridge University Press: Cambridge, 2012.

(41) Ghosh, S.; Chizhik, A. M.; Yang, G.; Karedla, N.; Gregor, I.; Oron, D.; Weiss, S.; Enderlein, J.; Chizhik, A. I. Excitation and Emission Transition Dipoles of Type-II Semiconductor Nanorods. Nano Lett. 2019, 19 (3), 1695-1700.

(42) Mertens, H.; Koenderink, A. F.; Polman, A. Plasmon-Enhanced Luminescence near Noble-Metal Nanospheres: Comparison of Exact Theory and an Improved Gersten and Nitzan Model. Phys. Rev. B: Condens. Matter Mater. Phys. 2007, 115123.

(43) Pellegrotti, J. V.; Acuna, G. P.; Puchkova, A.; Holzmeister, P.; Gietl, A.; Lalkens, B.; Stefani, F. D.; Tinnefeld, P. Controlled Reduction of Photobleaching in DNA Origami-Gold Nanoparticle Hybrids. Nano Lett. 2014, 14 (5), 2831-2836. 
(44) Rogobete, L.; Kaminski, F.; Agio, M.; Sandoghdar, V. Design of Plasmonic Nanoantennae for Enhancing Spontaneous Emission. Opt. Lett. 2007, 32, 1623.

(45) Blanco, L. A.; García de Abajo, F. J. Spontaneous Emission Enhancement near Nanoparticles. J. Quant. Spectrosc. Radiat. Transfer 2004, 89, 37.

(46) Busson, M. P.; Rolly, B.; Stout, B.; Bonod, N.; Bidault, S. Accelerated Single Photon Emission from Dye Molecule-Driven Nanoantennas Assembled on DNA. Nat. Commun. 2012, 1964.

(47) Liaw, J. W.; Chen, C. S.; Chen, J. H. Enhancement or Quenching Effect of Metallic Nanodimer on Spontaneous Emission. J. Quant. Spectrosc. Radiat. Transfer 2010, 111, 454.

(48) Mock, J. J.; Hill, R. T.; Degiron, A.; Zauscher, S.; Chilkoti, A.; Smith, D. R. Distance-Dependent Plasmon Resonant Coupling between a Gold Nanoparticle and Gold Film. Nano Lett. 2008, 8 (8), 2245-2252.

(49) Puchkova, A.; Vietz, C.; Pibiri, E.; Wünsch, B.; Sanz Paz, M.; Acuna, G. P.; Tinnefeld, P. DNA Origami Nanoantennas with over 5000-Fold Fluorescence Enhancement and Single-Molecule Detection at $25 \mathrm{Mm}$. Nano Lett. 2015, 15 (12), 8354-8359.

(50) Toscano, G.; Raza, S.; Jauho, A.-P.; Mortensen, N. A.; Wubs, M. Modified Field Enhancement in Plasmonic Nanowire Dimers Due to Nonlocal Response. Nat. Commun. 2011, 2 (1), 481.

(51) Yao, K.; Liu, Y. Controlling Electric and Magnetic Resonances for Ultracompact Nanoantennas with Tunable Directionality. ACS Photonics 2016, 3 (6), 953-963. 